\title{
Tunable Multi-functional Optofluidic Biconcave Lens
}

\author{
Chaolong Song, Trung-Dung Luong and Nam-Trung Nguyen \\ School of Mechanical and Aerospace Engineering \\ Nanyang Technological University \\ Singapore 639798
}

\begin{abstract}
Recently using the integrated micro-lens to improve the fluorescence detection in microfluidic systems has been intensively studied. This paper describes the design and demonstration of an optofluidic in-plane bi-concave lens to perform both light focusing and diverging. In both focusing and diverging mode, the optical properties of the lens can be tuned by adjusting the flow rate. Numerical simulation and experiment are conducted and the results are compared. Our device shows a good tunability.
\end{abstract}

\section{INTRODUCTION}

Fluorescence has been widely used in biochemistry and analytical chemistry as a non-destructive mean of tracking or analysis of bio-molecules or chemicals. Traditionally, the excitation of fluorescence is introduced by employing an external mercury lamp with a filter set or a laser with a specific wavelength. Recently, the emergence of microfluidics leads to the realization of on-chip biological/chemical assays. Fluorescence excitation and detection have also been integrated into a chip to make the whole system cost-effective and portable [1]. To improve the performance of the fluorescence excitation and to modify the light properties, many efforts have been devoted to integrate in-plane solid or liquid (fluidic) lens into microfluidic network [2], [3], [4]. Currently, most of the in-plane light modifications only involve a tuneable light focusing along the optical axis. This paper describes the design and demonstration of an optofluidic bi-concave lens that can perform both light focusing and diverging. Combining the performance of focusing and diverging, our device can provide multiple lens-effects to modify the in-plane light and therefore make the way of fluorescence excitation more flexible.

\section{DEVICE CONCEPT AND NUMERICAL MODELLING}

In traditional manufacturing of optics, a concave lens is fabricated with glass, which has a refractive index (RI) higher than that of surrounding medium, and thus cannot be used to focus light. The properties of an optofluidic component can be changed simply by replacing the liquid inside the component [5]. In the focusing mode of our optofluidic concave lens, a liquid with low refractive index is used as the core stream and a liquid with higher refractive index serves as cladding stream illustrated in figure 1 (a). It is found that for a $\mathrm{L}^{2}$ lens system the mis-match of refractive index between cladding liquid and PDMS brings about light scattering [6], [7]. In this work, we introduce an auxiliary cladding liquid with a very low flow rate and a refractive index matching PDMS to prevent the light beam from scattering. The light emitted from an optical fibre can be well focused and the focal length can be tuned by adjusting the flow rate ratio between core and cladding streams. If the core inlet is blocked, the liquid from cladding inlets will converge into a new core stream and the liquid from the auxiliary cladding inlet will serve as cladding streams. In this way, our device can switch from focusing mode to diverging mode. In the diverging mode of our concave lens, a liquid with high refractive index works as core stream, and a liquid with refractive index matching PDMS is used as cladding streams (figure 1 (b)). Owing to the higher refractive index of core stream and the tuneable lens interface, the divergence of the light beam can be expanded and adjusted.

The optofluidic lens is hydrodynamically formed in a rectangular chamber measuring $1 \mathrm{~mm} \times 1 \mathrm{~mm}$, which has one core inlet, two pairs of cladding inlets and two outlets. The width of the inlets and outlets is $50 \mu \mathrm{m}$. To characterize the performance of the concave lens by ray tracing, an optical fibre is introduced by a pre-defined channel with a width of $130 \mu \mathrm{m}$. The distance between the tip of fibre and the center of the rectangular chamber is $4 \mathrm{~mm}$. An aperture with a size of $400 \mu \mathrm{m}$ is built by filling ink into two channels. A raytracing chamber filled with fluorescence dye is placed behind the rectangular chamber to visualize the propagation of light rays. A two-dimensional numerical model is built based on the above geometry of the test device. The level-set method is applied to treat the interfaces between different fluids [8]. The Navier-Stokes equations governing the fluids are solved on the staggered grid system by a finite volume method with SIMPLER algorithm [9]. The level-set and redistancing functions are implemented within a narrow-band region near the interface instead of the whole computational domain to reduce computational effort. The level-set equation is solved with the high order fast sweeping method [10]. The computed positions of the interface were transferred into Matlab (MathWorks, Inc.), where a program was coded to implement the numerical ray-tracing.

\section{EXPERIMENTAL RESULTS AND DISCUSSIONS}

Our test devices were fabricated in polydimethylsiloxane (PDMS) using the standard soft lithography technique. In the focusing mode test, ethanol ( $\mathrm{RI} n=1.36$; viscosity $\mu=1.2 \times$ $10^{-3} \mathrm{Ns} / \mathrm{m}^{2}$ ) was used as the core liquid, cinnamaldehyde 


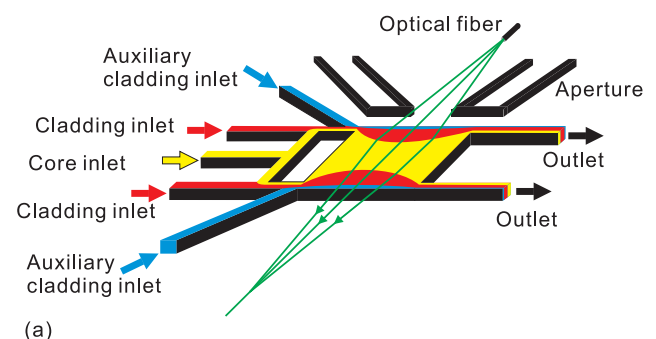

(a)
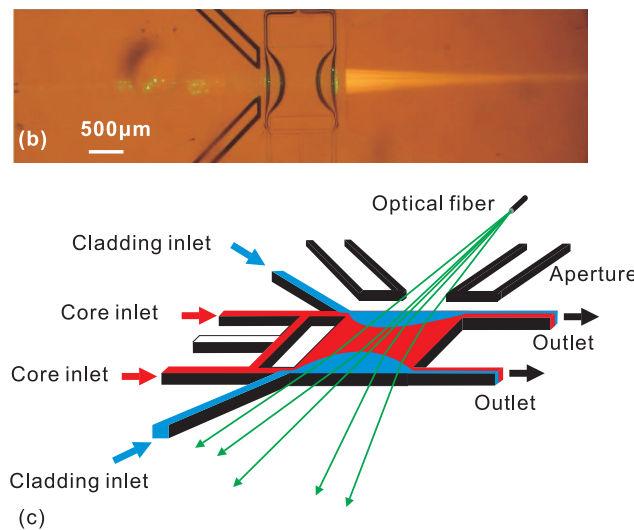

(c)

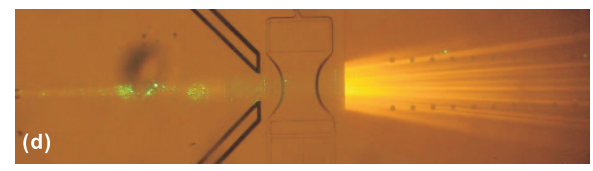

Fig. 1. Schematics: (a) Illustration of the optofluidic concave lens in the focusing mode; (b) Bright field image of the concave lens focusing a light beam; (c) Illustration of the concave lens in the diverging mode; (d) Bright field image of the concave lens spreading a light beam.

(RI $\mathrm{n}=1.62$; viscosity $\mu=5.7 \times 10^{-3} \mathrm{Ns} / \mathrm{m}^{2}$ ) as the cladding liquid, and a mixture of $73.5 \%$ ethylene glycol and $26.5 \%$ ethanol (viscosity $\mu=9.8 \times 10^{-3} \mathrm{Ns} / \mathrm{m}^{2}$ ) with a refractive index matching PDMS $(\mathrm{n}=1.412)$ worked as the auxiliary cladding liquid. In the diverging mode test, the pumping of core liquid was stopped to enable the liquid from the cladding inlets to converge into a new core stream (cinnamaldehyde), and therefore changing the role of the auxiliary cladding liquid from light-scattering protection to providing a mis-match of refractive index with that of the core stream to refract the light rays.

Figure 2 shows the manipulation of the lens interface by adjusting the flow rate ratio between core and cladding streams for the focusing mode of the optofluidic bi-concave lens. The error bars in figure 2, as well as in other figures, show the effect of flow fluctuation inside the rectangular chambers. During the test, the flow rate of the cladding streams and the auxiliary cladding stream were fixed at $1 \mathrm{~mL} / \mathrm{h}$ and 0.1 $\mathrm{mL} / \mathrm{h}$ respectively. The flow rate of the core stream was increased from $2 \mathrm{~mL} / \mathrm{h}$ to $14 \mathrm{~mL} / \mathrm{h}$. At low flow rate ratio $\left(\phi_{\text {core }} / \phi_{\text {cladding }}\right)$, the curvature of the interface between the core and cladding streams is small due to the relatively straight streamlines in the center part of the rectangular chamber. When the interface approaches the side-wall of the chamber at high flow rate ratio, the value of the curvature decreases owing

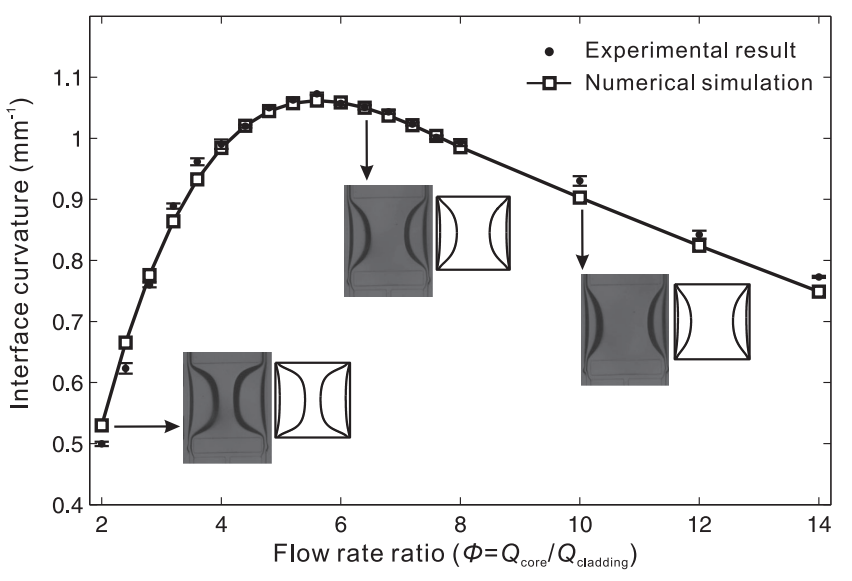

Fig. 2. Relationship between the flow rate ratio and the curvature of the interface in the focusing mode of the optofluidic bi-concave lens.

to the less curved streamlines near the boundary. When the position of the interface locates between the center and sidewall of the chamber, the curvature can achieve a maximum value. As a result of the significantly lower flow rate of the auxiliary cladding stream compared to the cladding streams, a thin layer liquid with a refractive index matching that of PDMS forms on the side-wall of the chamber. This thin layer prevents the scattering of the incident light on the concave lens that is due to the roughness of the PDMS surface, and the small thickness of the layer is supposed to reduce the refraction effect by this layer itself.

Ray tracing method was carried out experimentally and numerically to investigate the optical performance of the optofluidic concave lens in the focusing mode. In the numerical simulation, the shape of the interface between different streams was computed with level-set method and imported into a custom Matlab program which implements the ray tracing based on Snell's law. In the experiment, a green laser with a wavelength of $\lambda=532 \mathrm{~nm}$ was used to excite the fluorescence dye in the ray-tracing chamber, and the visualized light rays were recorded in gray-scale images. Due to the refractive index of the core liquid (ethanol $n=1.36$ ) being lower than that of the cladding liquid (cinnamaldehyde $\mathrm{n}=1.62$ ), the optofluidic concave lens possesses light focusing effect. The simulation and experimental results, which were obtained at flow rate ratio of 2, 6, and 10, are compared and shown in Figure 3. At the low flow rate ratio $\phi=2$, the concave lens has a longer focal length owing to the small curvature when the interface is positioned around the center part of the chamber, Fig. 3(a). By increasing the flow rate ratio, the interface becomes more curved, which results in a shorter focal length, Fig. 3(b). When the interface approaches the side-wall of the rectangular chamber, the curvature of the interface decreases, thus elongating the focal length, Fig. 3(c).

To demonstrate the importance of the auxiliary cladding stream in the focusing mode of the optofluidic bi-concave lens, we compare the optical performance of the concave lens with and without this stream. We carried out the testing at a 

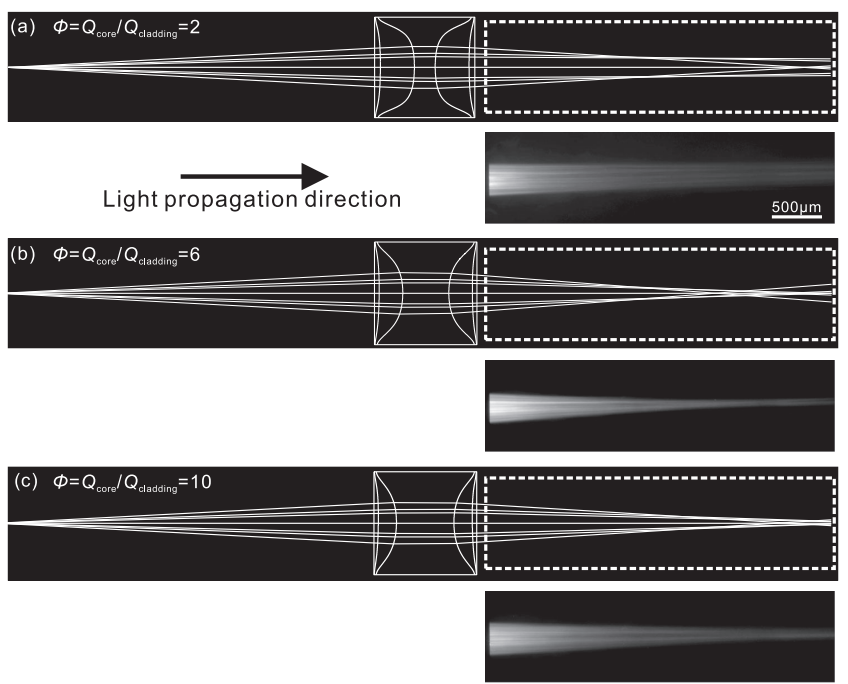

Fig. 3. Comparisons between experimental and numerical ray-tracing results under conditions of different flow rate ratios between core and cladding streams in the focusing mode.

flow rate ratio of 6 . The ray-tracing images were captured and shown in Fig. 4 (a) and (b). The intensity distributions along the focal plane were extracted and compared in Fig. 4 (c). The comparison shows that the concave lens with auxiliary cladding stream has a narrower beam width at the focal plane, and its intensity profile is more smoothly and tightly distributed. However, the beam width of the concave lens without the auxiliary cladding stream is wider, and the intensity profile is not continuously distributed. This phenomenon is due to the rough PDMS surface, which would diffusively refract the light rays when the refractive index of cladding stream is different from that of PDMS.

The focusing mode can be simply converted to diverging mode by stopping the pumping of liquid (ethanol) from the core inlet. By this way, the previous cladding liquid (cinnamaldehyde) in focusing mode will converge in the rectangular chamber and become a core stream in the diverging mode, and the role of the auxiliary cladding liquid (mixture of ethylene glycol and ethanol) changes from preventing lightscattering to refracting light rays (Fig. 1 (c)). Therefore, the core and cladding liquids involved in the diverging mode testing are cinnamaldehyde and the mixture of $73.5 \%$ ethylene glycol and $26.5 \%$ ethanol respectively. Figure 5 shows the relationship between the flow rate ratio ( $\left.\phi_{\text {core }} / \phi_{\text {cladding }}\right)$ and the curvature of the interface for the diverging mode. In the test, the core stream was pumped with a fixed flow rate 2 $\mathrm{mL} / \mathrm{h}$, and the flow rate of cladding stream was varied from $0.2 \mathrm{~mL} / \mathrm{h}$ to $5 \mathrm{~mL} / \mathrm{h}$. Because the streamlines at the center and near the side-wall of the rectangular chamber have relatively straight line shape, the values of the curvature of the interface at low and high flow rate ratios are smaller. And when the interface is positioned between the center and the side-wall of the chamber, the curvature can achieve a maximum value. This trend of the curve is similar to that in the focusing mode (a)

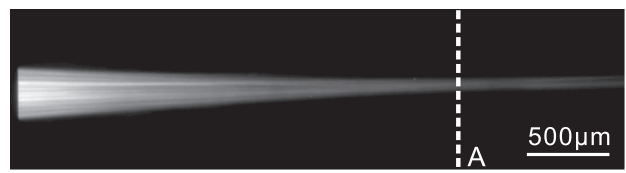

(b)
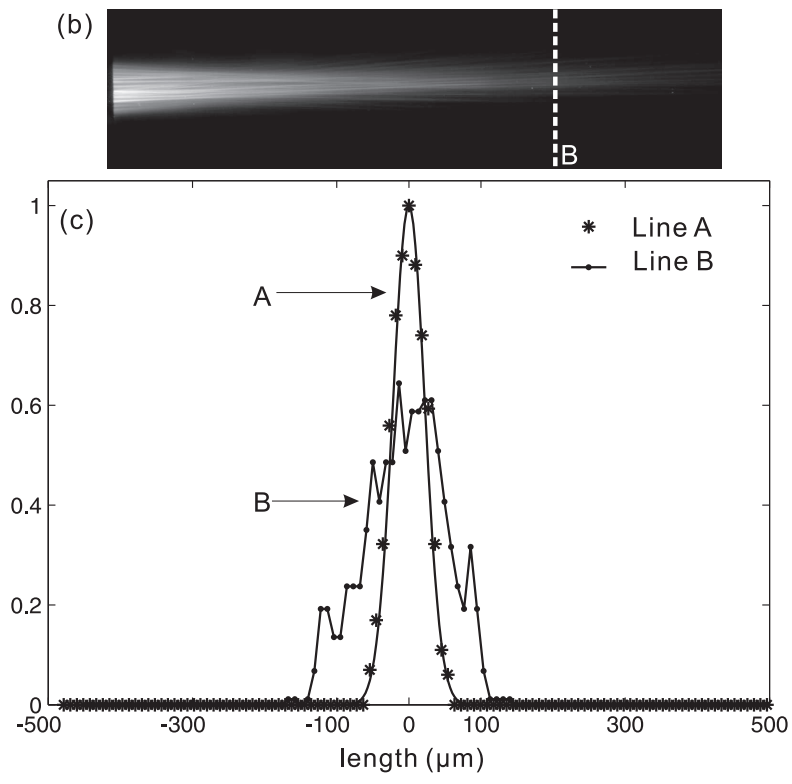

Fig. 4. Comparison between the optofluidic bi-concave lens with and without the auxiliary cladding streams in the focusing mode. The light beam is focused under a condition of flow rate ratio 6. (a) A well focused light beam by a concave lens with the auxiliary cladding stream; (b) a focused and scattered light beam by a concave lens without the auxiliary cladding stream; (c) intensity profiles extracted from the gray-scale images (a) and (b).

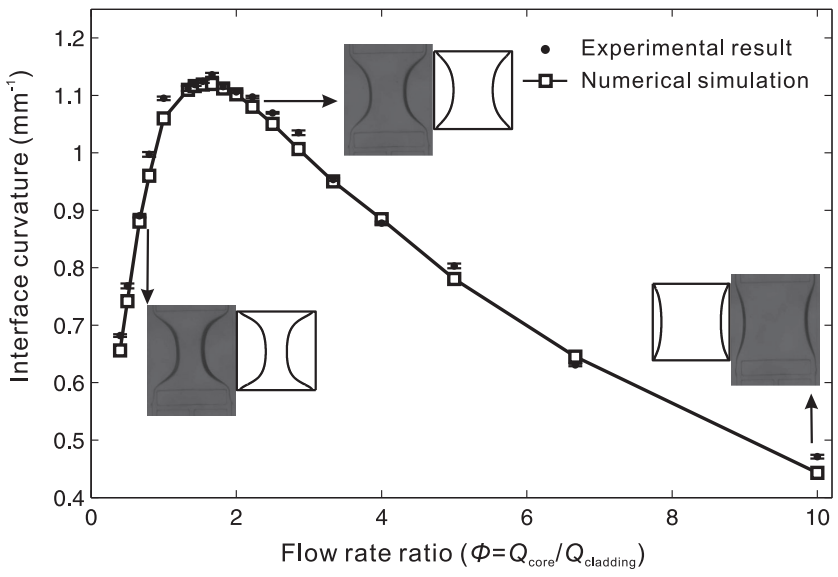

Fig. 5. Relationship between the flow rate ratio and the curvature of the interface in the diverging mode of the optofluidic bi-concave lens.

testing.

Due to the higher refractive index of the core liquid in the diverging mode, the optofluidic bi-concave lens spreads the light rays thus expanding the divergent angle of the light beam originating from the optical fiber. To investigate the optical performance of this concave lens, we have also implemented experimental and numerical ray-tracing method in the same way as the investigation of the focusing mode. The comparison between experimental results and numerical 
simulation is illustrated in figure 6 . The results were obtained at flow rate ratios of 20,10,2 and 0.5. At the highest flow rate ratio of 20 , the interface is close to the side-wall of the chamber resulting in the small curvature of the interface having little impact on the refraction of light rays. Therefore the divergent angle of the light beam from the fiber remains almost unchanged after passing the concave lens. With a decrease in the flow rate ratio, the curvature of the interface is increased, so the concave lens greatly expands the divergent angle of the light beam. However, as the interface approaches the center of the chamber, its curvature drops, and the divergent angle of the light beam reduces as well.

\section{CONCLUSION}

In this paper, we reported the design and characterization of an optofluidic bi-concave lens, which can perform both light focusing and diverging. In the focusing mode of the concave lens, a liquid (ethanol) with low refractive index is used as the core stream and a liquid (cinnamaldehyde) with higher refractive index serves as cladding stream. The relatively lower refractive index of core and the concave shape of the interface conjunctly enable the lens to focus light. An auxiliary cladding liquid with refractive index matching PDMS was introduced to prevent the light rays from scattering at the rough surface of the chamber wall. To switch the concave lens from focusing mode to diverging mode, the flow of the core liquid (ethanol) was stopped. And the previous cladding liquid (cinnamaldehyde) would join together in the chamber and become the core stream for the diverging mode. The auxiliary cladding liquid would change its function from avoiding lightscattering to becoming the cladding stream for the diverging mode. The testing of our device as well as the numerical analysis demonstrates that our optofluidic bi-concave lens has a multi-function allowing both focusing and diverging a light beam.

\section{REFERENCES}

[1] M. L. Chabinyc, D.T. Chiu, J.C. McDonald, A.D. Stroock, J.F. Christian, A.M. Karger, and G.M. Whitesides, "An integrated fluorescence detection system in poly(dimethylsiloxane) for microfluidic applications," Analytical Chemistry, vol. 73, pp. 4491-4498, 2001.

[2] S. Camou, H. Fujita, and T. Fujii, "PDMS 2D optical lens integrated with microfluidic channels: Principle and characterization," Lab on a Chip Miniaturisation for Chemistry and Biology, vol. 3, pp. 40-45, 2003.

[3] S.K.Y. Tang, C.A. Stan, and G.M. Whitesides, "Dynamically reconfigurable liquid-core liquid-cladding lens in a microfluidic channel," Lab on a Chip - Miniaturisation for Chemistry and Biology, vol. 8, pp. 395-401, 2008.

[4] C. Song, N.T. Nguyen, S.H. Tan, and A.K. Asundi, "Modelling and optimization of micro optofluidic lenses," Lab on a Chip - Miniaturisation for Chemistry and Biology, vol. 9, pp. 1178-1184, 2009.

[5] D. Psaltis, S.R. Quake, and C. Yang, "Developing optofluidic technology through the fusion of microfluidics and optics," Nature, vol. 442, pp. 381-386, 2006.

[6] C. Song, N.T. Nguyen, S.H. Tan, and A.K. Asundi, "A tuneable microoptofluidic biconvex lens with mathematically predictable focal length," Microfluidics and Nanofluidics, vol. 9, pp. 889-896, 2010.

[7] C. Song, N.T. Nguyen, A.K. Asundi, and C.L.N. Low, "Biconcave microoptofluidic lens with low-refractive-index liquids," Optics Letter, vol. 34, pp. 3622-3624, 2009

[8] S. Osher and J.A. Sethian, "Fronts propagating with curvature-dependent speed: Algorithms based on Hamilton-Jacobi formulations," Journal of Computational Physics, vol. 79, pp. 12-49, 1988.
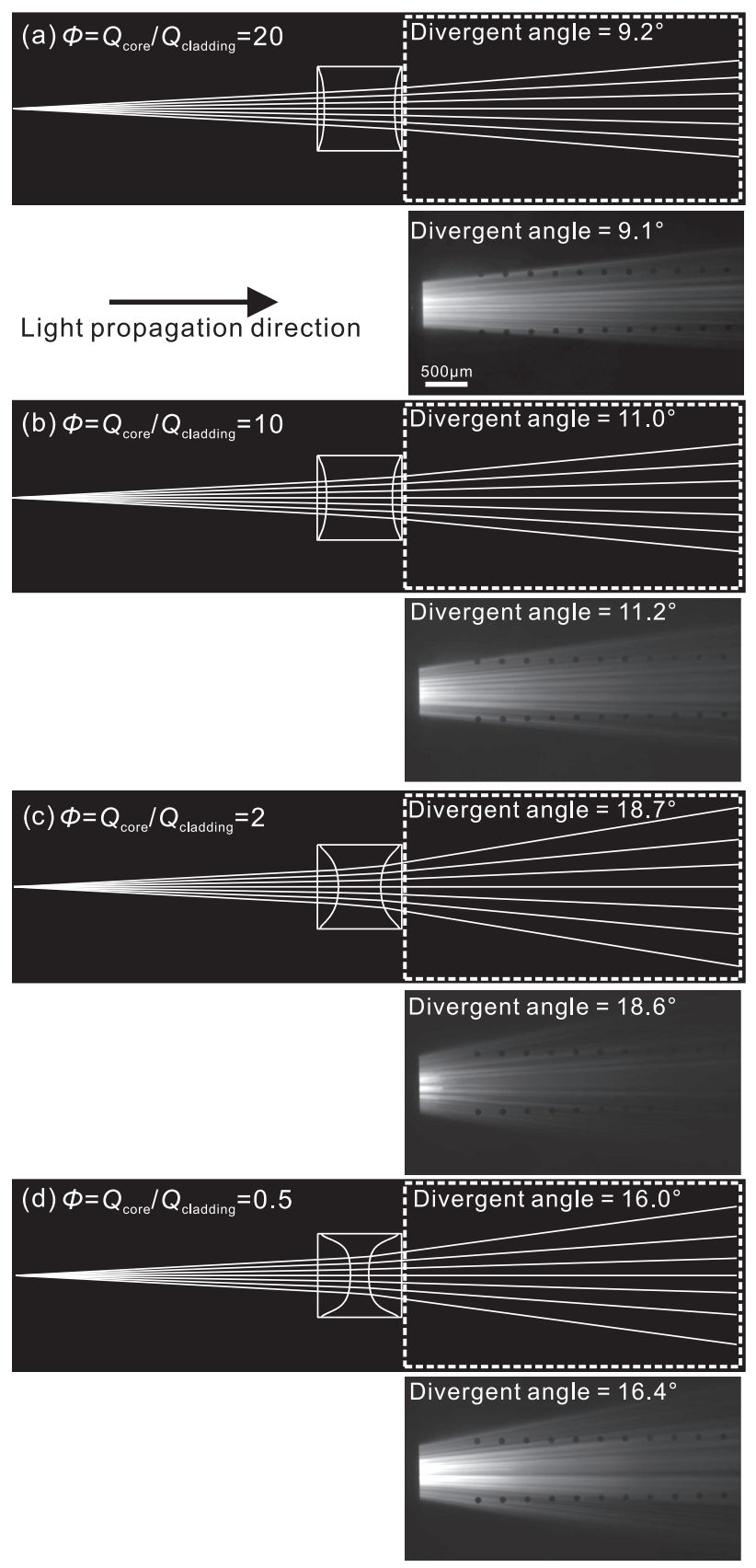

Fig. 6. Comparisons between experimental and numerical ray-tracing results under conditions of different flow rate ratios between core and cladding streams in the diverging mode.

[9] J. U. Patankar, Numerical Heat Transfer and Fluid Flow, Hemisphere, New York, 1980.

[10] Y. T. Zhang, H.K. Zhao, and J. Qian, "High order fast sweeping methods for static Hamilton-Jacobi equations," Journal of Scientific Computing, vol. 29 , pp. $25-56,2006$ 